\title{
Inhibition of mesenchymal stromal cells by pre-activated lymphocytes and their culture media
}

\author{
Erica Valencic ${ }^{1 \dagger}$, Claudia Loganes ${ }^{1 \dagger}$, Stefania Cesana ${ }^{2,3}$, Elisa Piscianz ${ }^{1}$, Giuseppe Gaipa ${ }^{2,3}$, Ettore Biagi ${ }^{2,3}$ \\ and Alberto Tommasini ${ }^{1 *}$
}

\begin{abstract}
Introduction: Despite having a proven immunosuppressive potential in vitro, human mesenchymal stromal cells (MSCs) are reported to display variable efficacy in vivo and, in fact, their proven benefit in the clinical practice is still limited and controversial.

Methods: The interplay between clinical grade MSCs and pre-activated donor lymphocytes or selected lymphocyte subsets was studied in vitro. The kinetics of MSC growth and viability was evaluated by adhesion-dependent changes of culture plate impedance and biochemically by a colorimetric assay. Activation of natural killer (NK) cells was assessed as well, using a flow cytometry assay.

Results: A strong inhibition of MSC growth was rapidly induced by the addition of pre-activated lymphocytes but not of resting lymphocytes. Inhibition seems not to be attributable to a single cell population, as similar results can be obtained by depleting NK cells or by using either selected $\mathrm{CD}^{+}{ }^{+}$or $\mathrm{CD}^{+}{ }^{+}$lymphocytes. In addition, conditioned medium (CM) from activated lymphocytes was able to inhibit MSC growth in a dose-dependent manner. Furthermore, licensing with IFN- $\gamma$ partially protected MSCs from pre-activated lymphocytes but not from their CM. These results suggest an inhibitory role of lymphocyte-activation-derived substances. However, the identification of a single molecule responsible for MSC inhibition remained elusive, even if preliminary experiments showed that ATP and, to a lesser extent, TNF-a might play a role.

Conclusions: These results suggest that survival of MSCs can be affected by soluble mediators released by activated lymphocytes. Thus it can be hypothesized that MSC immunosuppressive action in vivo could be impaired by ongoing immune activation through the release of inflammatory mediators.
\end{abstract}

\section{Introduction}

Mesenchymal stromal cells (MSCs) are a heterogeneous population of cells that can be obtained from bone marrow and from other adult tissues and are able to proliferate in vitro as plastic-adherent fibroblast-like cells [1-7]. In vitro, they have a potent immunosuppressive action that justified their use in clinical trials for the treatment of steroid-resistant graft versus host disease (GvHD) and for severe autoimmune diseases. In fact, MSCs are thought to be poorly immunogenic and have been shown to have a highly safe profile in vivo. However, the results of clinical trials so far showed heterogeneous

\footnotetext{
* Correspondence: alberto.tommasini@burlo.trieste.it

${ }^{\dagger}$ Equal contributors

'Department of Pediatrics, Institute of Maternal and Child Health IRCCS Burlo Garofolo, Via dell'Istria 65/1, 34137 Trieste, Italy

Full list of author information is available at the end of the article
}

efficacy [8-11], and contradictory results have been published about their immunogenicity [12-14]. In particular, it is becoming evident that microenvironmental factors may play a major role in conditioning the immunosuppressive action of MSCs. On one hand, natural killer (NK) cells are able to kill MSCs in appropriate conditions [15]. On the other hand, the immunosuppressive potency of MSCs could be increased in vitro by their exposure to exogenous molecules, such as lymphocyte-derived cytokines, such as interferon-gamma (IFN- $\gamma$ ). For example, MSCs that have been previously exposed in vitro to IFN- $\gamma$ are resistant to NK-cell-mediated lysis, maybe due to an up-regulation of class 1 human leukocyte antigen (HLA-I) on their surface $[15,16]$. As a matter of fact, pre-clinical results may vary substantially according to the setting of the co-culture between lymphocytes and MSCs. We have previously shown that whether lymphocytes are activated before or during

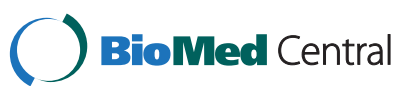


incubation with MSCs and, the other way around, whether MSCs are treated or not with licensing-substances before the co-culture are both critical factors influencing the outcome of immunosuppression.

In our model, the stimulation of lymphocytes before incubation with MSCs is meant to mimic the GvHD condition in vivo, considering that the therapeutic use of MSCs is proposed for patients with already active immune responses. In this model, pre-activated lymphocytes are resistant to the action of MSCs, unless MSCs have been previously exposed to IFN- $\gamma$ [17].

In the present study, to unravel better the interactions between lymphocytes and MSCs, we used a non-invasive tool for real-time monitoring of the vitality and growth of MSCs after the addition of peripheral blood mononuclear cells (PBMCs) [18].

\section{Methods}

\section{Cell culture}

Human bone marrow MSCs were obtained at the Laboratory for Cell and Gene Therapy 'S Verri', Monza, Italy [11]. Side collections of clinical grade MSC preparations were dedicated to this research, after obtaining the donors' informed consent. The research plan was approved by the Institute Research Board (reference number \#RC 32/11) and by the local ethics committee at the IRCCS Burlo Garofolo, Trieste, Italy.

In brief, total nucleated cells were isolated from the washouts of discarded bone marrow collection bags and filters by washing both the filter and the empty bag twice with phosphate-buffered saline and centrifuging at 1,600 rpm for 5 minutes. The cells were plated at 500,000 cells $/ \mathrm{cm}^{2}$ in Dulbecco's modified essential medium low glucose (Gibco-Invitrogen, Carlbad, CA, USA), supplemented with $2 \mathrm{UI} / \mathrm{ml}$ heparin (Pharmatex, Milan, Italy) and $5 \%$ platelet lysate (hPL) and cultured in $5 \% \mathrm{CO}_{2}$ at $37^{\circ} \mathrm{C}$. Non-adherent cells were removed after two to three days and a 50\% medium change was performed twice weekly until $80 \%$ confluence. Cells were recovered by trypsinization, re-plated at $200 \mathrm{cells} / \mathrm{cm}^{2}$ and cultured for an additional 12 days. After trypsinization, the cells were frozen in vials in $10 \%$ clinical grade dimethyl sulfoxide (DMSO) (WAK-Chemie Medica GmbH, Steinbach, Germany) and $90 \%$ human $\mathrm{AB}$ plasma.

When ready to be used, the cells were thawed and plated in T75 flask (Falcon, BD Biosciences, Bedford, MA USA) at 8,000 cells $/ \mathrm{cm}^{2}$ in Dulbecco's modified essential medium low glucose (Euroclone, Milan, Italy), supplemented with $100 \mathrm{U} / \mathrm{ml}$ penicillin, $100 \mu \mathrm{g} / \mathrm{ml}$ streptomycin, $200 \mathrm{mM}$ L-glutamine (Euroclone), 5\% platelet lysate (called complete medium), and cultured in $5 \% \mathrm{CO}_{2}$ at $37^{\circ} \mathrm{C}$. Following trypsinization, at the third passage, the cells were seeded in a 16-well electric microtiter plate for the co-culture experiments.
PBMCs were isolated from adult healthy volunteers, after obtaining their informed consent according to the local ethical committee, by centrifugation on biocoll separating solution (Biochrom AG, Berlin, Germany) at $560 \times \mathrm{g}$ for 30 minutes at room temperature. $\mathrm{CD}^{+}$ and $\mathrm{CD}^{+}$cells were then separated by negative selection using magnetic sorting (Miltenyi Biotec, Bergisch Gladbach, Germany). PBMCs depleted of $\mathrm{CD}^{+} 6^{+}$cells were obtained using anti-CD56 coated beads (Miltenyi Biotec).

PBMC, $\mathrm{CD}^{+}$cells, $\mathrm{CD} 8^{+}$cells, CD56-depleted PBMCs were stimulated or not with $1 \mu \mathrm{g} / \mathrm{ml}$ phytohemagglutinin (PHA) (Sigma Aldrich, Milan, Italy) 24 hours before the co-culture and used as effector cells to test how they affect MSC growth.

In a new set of experiments, MSCs were cultivated in the presence of conditioned medium (CM) from $1 \times 10^{6}$ PBMCs activated with $1 \mu \mathrm{g} / \mathrm{ml}$ PHA or with anti CD3/ CD28 beads (Dynabeads ${ }^{\circ}$ Human T-activator CD3/CD28, Invitrogen Dynal AS, Oslo, Norway) in complete medium for 24 hours.

CM from PBMCs, activated or not with PHA, were assessed by means of a bead-based multiplex immunoassay (27 human-Bio-Plex assay; BioRad Laboratories, Milan, Italy), following the manufacturer's instructions. Human cytokine and chemokine levels were measured in duplicate, using the Bio-Plex 200 reader (Bio-Rad, Hercules, CA, USA) equipped with Bioplex Manager 6 software.

\section{Co-culture experiments with impedance measurements}

Measurements were carried out with an xCELLigence RTCA DP instrument (Roche, Penzberg, Germany). In this instrument, the presence of adherent cells on the bottom of the plate leads to an increase of electrode impedance, which is converted in a 'Cell Index' (CI) that depends on the number and viability of attached cells. Thus, the death and detachment of cultured cells will be recorded as a decrease in impedance. In this 16-well device (E-Plate 16) $400 \mathrm{MSCs} /$ wells were plated in $200 \mu \mathrm{l}$ of complete medium and the impedance was measured every five minutes.

Cells were allowed to attach and proliferate. When they had reached their growth phase, as evidenced by curve profiles, $100 \mu \mathrm{l}$ of medium were removed and PBMCs were added at varying cell ratios to seeded MSCs or CM was added at varying dilutions.

In a set of experiments, attached MSCs were pre-treated with IFN- $\gamma$ (at a final concentration of $1,000 \mathrm{U} / \mathrm{ml}$ ), 24 hours before the stimulation.

This impedance assay was reproduced four times with different MSC populations, treated with PBMCs (or their $\mathrm{CM})$ from various healthy volunteers. Each experiment used a single donor's cells and was conducted in triplicate. 


\section{NK degranulation assay}

The ability of MSCs to stimulate NK cells was investigated by flow cytometry, by measuring the MSC-induced NK-degranulation as revealed by CD107a surface expression [19]. Briefly, $2 \times 10^{5}$ donor PBMCs (resting or PHA-activated) and $2 \times 10^{5}$ MSCs were co-cultured in suspension in the presence of anti-CD107a-PE (BD Biosciences, San Jose, CA, USA). An equal number of PBMCs was incubated with $2 \times 10^{5} \mathrm{~K} 562$ tumor cell line (ATCC CCL 243), as positive control of degranulation, or with medium alone (Iscove's modified Dulbecco's medium, IMDM), as negative control. After two hours of incubation at $37^{\circ} \mathrm{C}$ and $5 \% \mathrm{CO}_{2}$ cells were harvested and stained with CD3-PerCP and CD56 APC (both from BD Biosciences). Data were acquired on a CyAn ADP flow cytometer (Beckman Coulter, Fort Collins, CO, USA) and analyzed using FlowJo software v 7.6 (TreeStar, Ashland, OR, USA). The expression of CD107a was recorded on $\mathrm{CD}^{-} \mathrm{CD}^{+} 6^{+}$cell gate.

\section{MTT cell viability assay}

Cell viability was evaluated biochemically with the MTT (3-(4,5-dimethylthiazol-2-yl)-2,5-diphenyltetrazolium bromide) method, after MSC treatment with different volumes of resting or pre-activated lymphocyte CM.

The assay was carried out under the same experimental conditions as those adopted for the xCELLigence protocol: $400 \mathrm{MSCs} /$ well were seeded in a 96-well plate in $200 \mu \mathrm{L}$ of complete medium; cells were allowed to proliferate and were treated, after about 72 hours, with lymphocyte $\mathrm{CM}$ at varying dilutions. Control wells were filled with medium alone to provide the blanks for absorbance readings and untreated control cells were included in the assay. To best match the results of xCELLigence kinetics, the MTT assay was repeated at different time points, 24, 48 and 72 hours after the addition of CM; at these time points, $20 \mu \mathrm{l}$ MTT (5 $\mathrm{mg} / \mathrm{ml}$ in PBS) were added to each well, including controls, and the culture plate returned to the cell culture incubator for four hours $\left(37^{\circ} \mathrm{C}, 5 \% \mathrm{CO}_{2}\right)$. Once incubation ended, the medium was carefully removed and $100 \mu \mathrm{l}$ DMSO were added to dissolve the intracellular punctuate purple precipitate that is proportional to the number of viable living cells. The absorbance was read at $590 \mathrm{~nm}$ with a reference filter of $620 \mathrm{~nm}$.

The extent of MTT conversion in treated cells was expressed as a percentage of the viability of the control cells. Each experiment was reproduced three times in duplicate.

\section{Statistical analysis}

Analyses were performed using GraphPad Prism v5c software. Data were evaluated by one-way analysis of variance (ANOVA) and by Student's t test.
The study was approved by the Institution Research Board and by the Independent Committee for Bioethics of the Institute Burlo Garofolo, with reference number $32 / 11$.

\section{Results}

Unstimulated PBMCs had little, if any, effect on the MSC CI. Only at the highest PBMC:MSC ratio (24:1), could a relevant inhibition of MSC growth be observed, as evidenced by a halving of the CI (from a CI of 0.656 to 0.327 ) (Figure $1 \mathrm{~A}$ and $\mathrm{C}$ ).

On the contrary, pre-stimulated PBMCs induced a strong decrease in MSC CI at all ratios, leading in all cases to a complete inhibition of proliferation (CI from 1.11 to 0.054 for $1: 3$ ratio and 0 for higher ratios). The decrease in MSC CI started in less than an hour after the addition of activated lymphocytes and was faster at higher PBMC:MSC ratios. Lower ratios of PBMCs seemed to exert a delayed effect, as a complete inhibition of MSC growth could be reached in two to three days, suggesting that they can continue proliferating and secreting cytokines during the incubation with MSCs (Figure 1B and C).

The inhibitory capacity of PBMCs on MSCs is retained after depletion of NK cells (Figure 2). This result is in agreement with the observation that MSCs were not able to induce NK activation compared to a conventional tumor cell line, as demonstrated by a CD107a expression similar to the negative control (Figure 3 ). Furthermore, single lymphocyte subsets $\left(\mathrm{CD} 4^{+}\right.$or $\left.\mathrm{CD} 8^{+}\right)$ maintained a strong inhibitory capacity on MSCs, which was evident at a 24:1 ratio. However, the effect of $\mathrm{CD} 4^{+}$ lymphocytes seemed to be more rapid if compared to $\mathrm{CD}^{+}$cells (data not shown).

A subsequent set of experiments was carried out to analyze the role of lymphocyte-derived soluble mediators on MSC growth. The addition of CM from both PHA-activated lymphocytes and antiCD3/CD28-activated lymphocytes inhibited the growth of MSCs in a dose-dependent manner (Figure $4 \mathrm{~B}$ and $\mathrm{C}$ for PHAactivated lymphocytes; Additional file 1: Figure A1 for CD3/CD28-activated lymphocytes). Compared to lymphocytes, CM induced a slower decrease in MSC CI. Moreover, only the highest concentration of CM produced a long lasting inhibition of growth, while a resumption of cell growth occurred during the last day of incubation in the presence of lower concentrations of CM.

Cytokine concentration was assessed in PHA-activated lymphocyte $\mathrm{CM}$, showing high levels of macrophage inflammatory protein-1 $\beta$ (MIP-1 $\beta$ ), interferon-inducible protein-10 (IP-10), TNF- $\alpha$, IFN- $\gamma$ and IL-2 (Figure 5).

As we have previously shown, licensed MSCs are partially resistant to the action of pre-activated lymphocytes, probably because they are able to block lymphocyte 

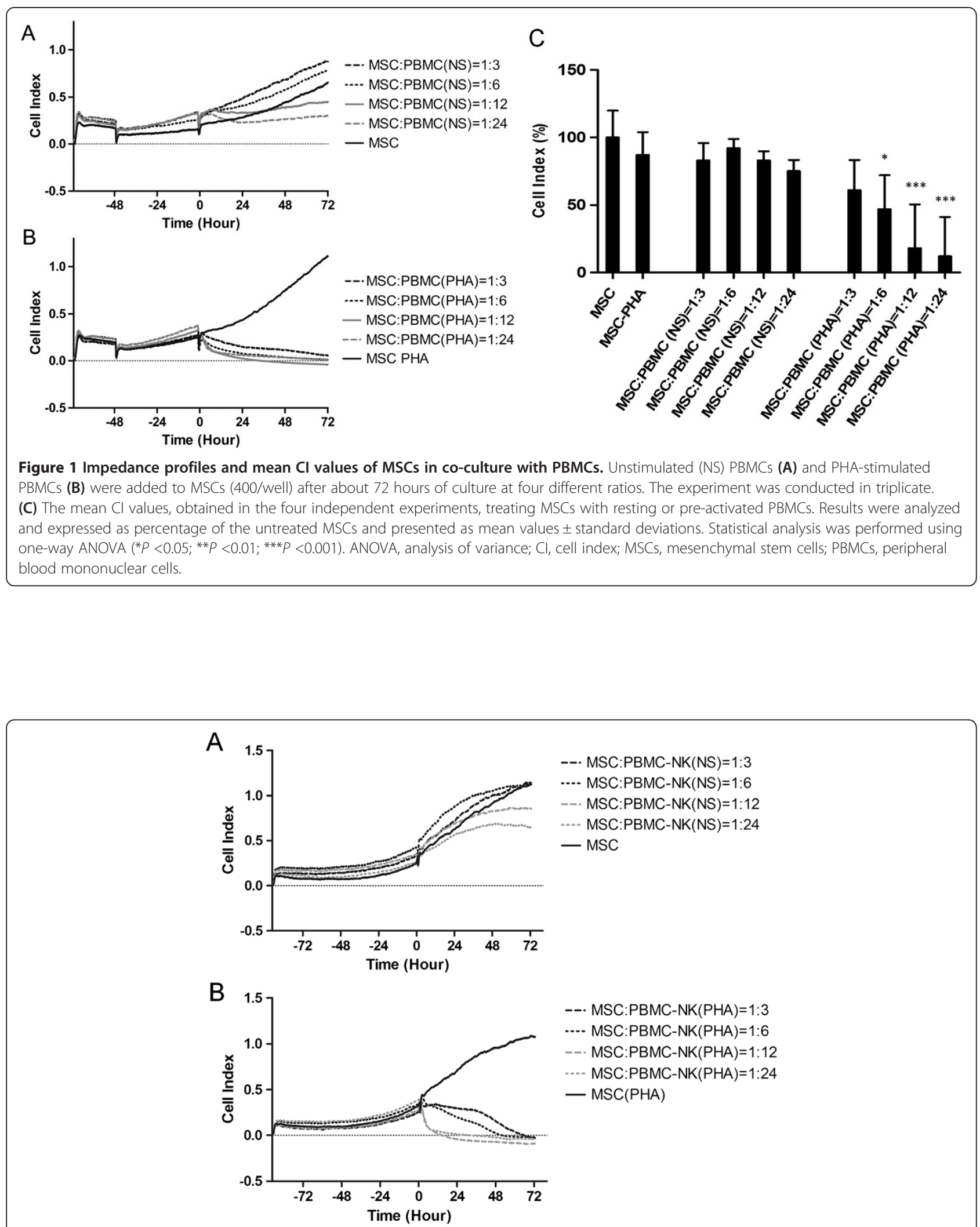

\section{--. MSC:PBMC-NK(PHA)=1:3 \\ .... MSC:PBMC-NK(PHA) $=1: 6$ \\ --. MSC:PBMC-NK(PHA)=1:12 \\ -... MSC:PBMC-NK(PHA) $=1: 24$ \\ - MSC(PHA)}

Figure 2 Impedance profiles of MSCs in co-culture with NK-depleted PBMCs. Unstimulated (NS) NK-depleted PBMCs (A) and PHA-stimulated NK-depleted PBMCs (B) were added to MSCs (400/well), after about 72 hours of culture, at four different ratios. The experiment was conducted in triplicate. MSCs, mesenchymal stem cells; NK, natural killer; PBMCs, peripheral blood mononuclear cells; PHA, phytohemagglutinin. 


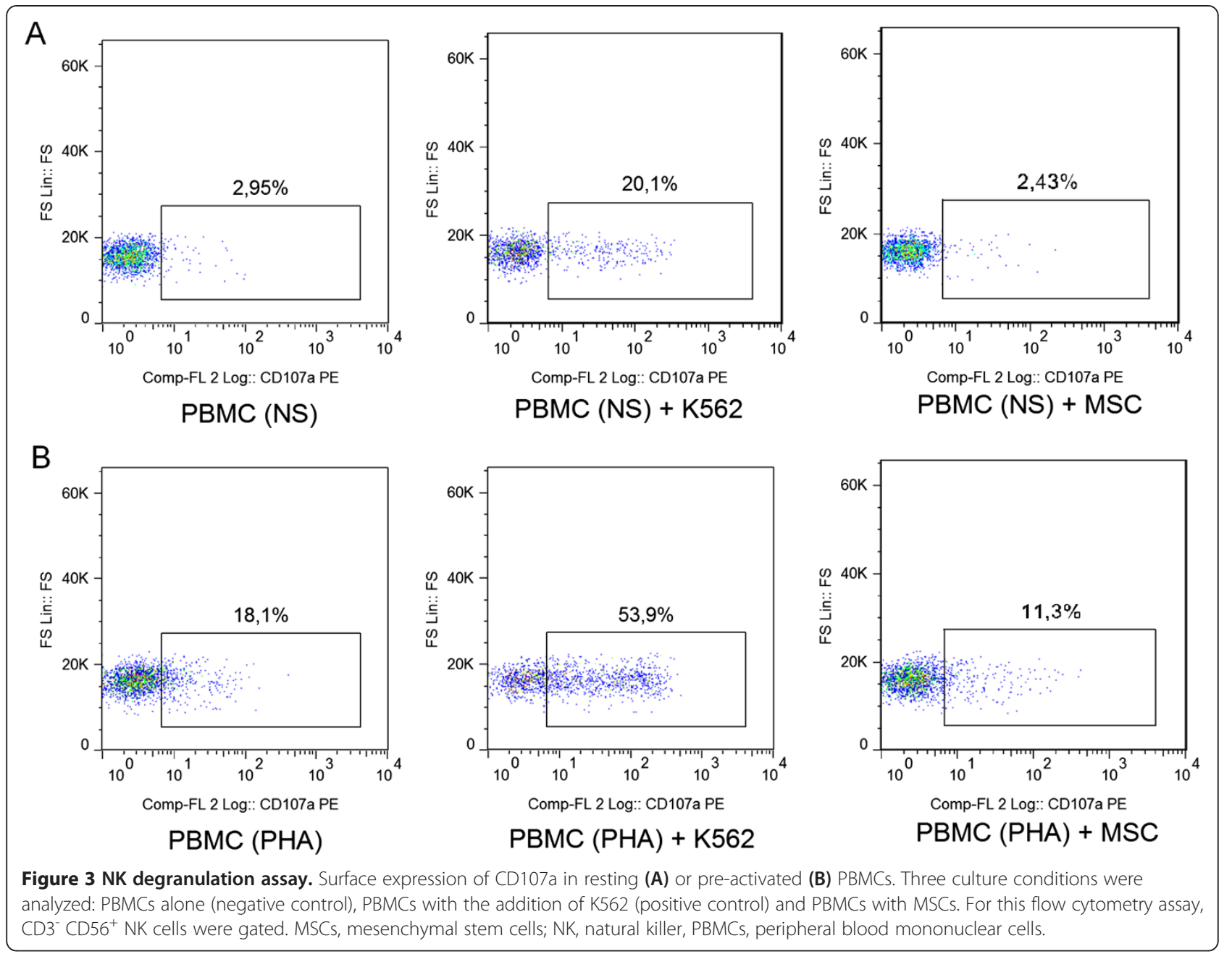

activation and proliferation before becoming themselves a target of lymphocytes [17]. In contrast, pre-exposure to IFN- $\gamma$ failed to protect MSCs from the action of CM (see Additional file 1: Figure A2 and A3).

The diminution of MSC CI after the addition of lymphocyte $\mathrm{CM}$ was paralleled by a diminution in the number of viable cells as assessed by the MTT assay; in particular, the experiment revealed that CM of pre-activated lymphocytes led to a progressive dose-dependent reduction in MSC viability, that was statistically significant only at the higher dose of CM $(50 \mu \mathrm{l}$ and $100 \mu \mathrm{l}), 48$ hours and 72 hours post-stimulation (Figure 6).

\section{Discussion}

Although the immunosuppressive potential of MSCs has been consistently demonstrated in vitro, the use of these cells in the clinical setting of immune disorders has remained controversial. Few controlled trials have been conducted which showed a highly variable efficacy in different patients [8-10]. The cause of such variability seems not to be attributable to the characteristics of
MSCs themselves or to HLA matching between donor and recipient, thus suggesting that the immune activation profiles of each single patient could influence the action of MSCs. Based on these observations, we argued that in vitro studies of the immunosuppressive action of MSCs should be carried out by using pre-stimulated lymphocytes, that is, lymphocytes that are already activated when MSCs are added in co-culture. In fact, this condition may better reflect the state of immune activation found in clinical situations, where the therapeutic use of MSCs is proposed [17]. We have previously shown that MSCs are not able to kill pre-stimulated lymphocytes unless they are pre-treated with IFN- $\gamma$ (17). Here, we analyzed the effect of activated lymphocytes on MSCs by using a new method based on the measure of electric impedance by means of xCELLigence instrumentation, allowing us to calculate a $\mathrm{CI}$ that reflects both growth and viability of adherent MSCs. Although plate impedance changes are only indirect measures of cell growth and death, other researchers have shown a very good correlation of impedance data with those 

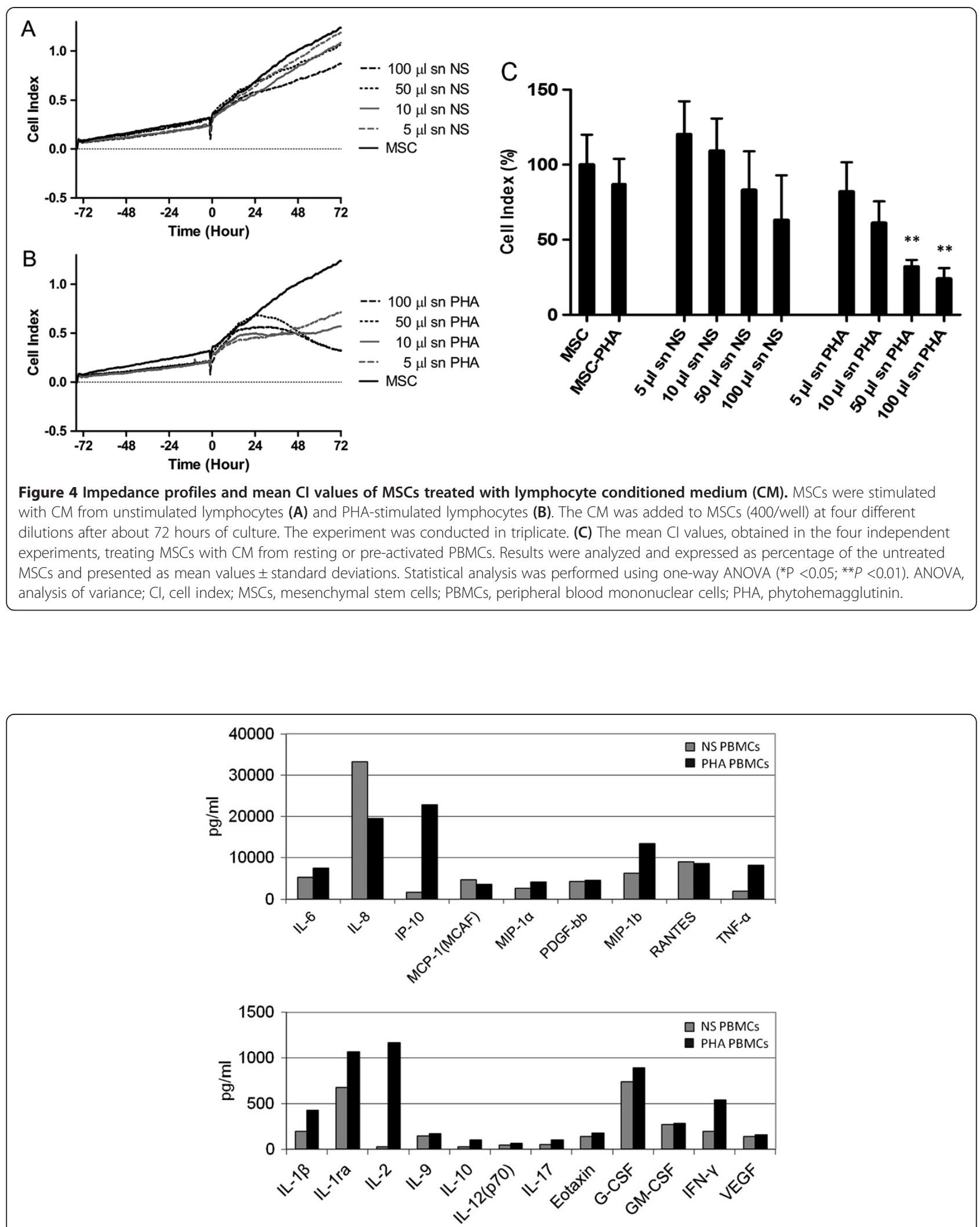

Figure 5 Measure of different cytokines in the conditioned medium of activated-lymphocytes. Values are expressed in pg/ml. Non-stimulated lymphocytes: grey bars; lymphocytes stimulated for 24 hours with $1 \mathrm{ug} / \mathrm{ml} \mathrm{PHA}$ : black bars. The cytokine quantification was conducted in duplicate. PHA, phytohemagglutinin. 


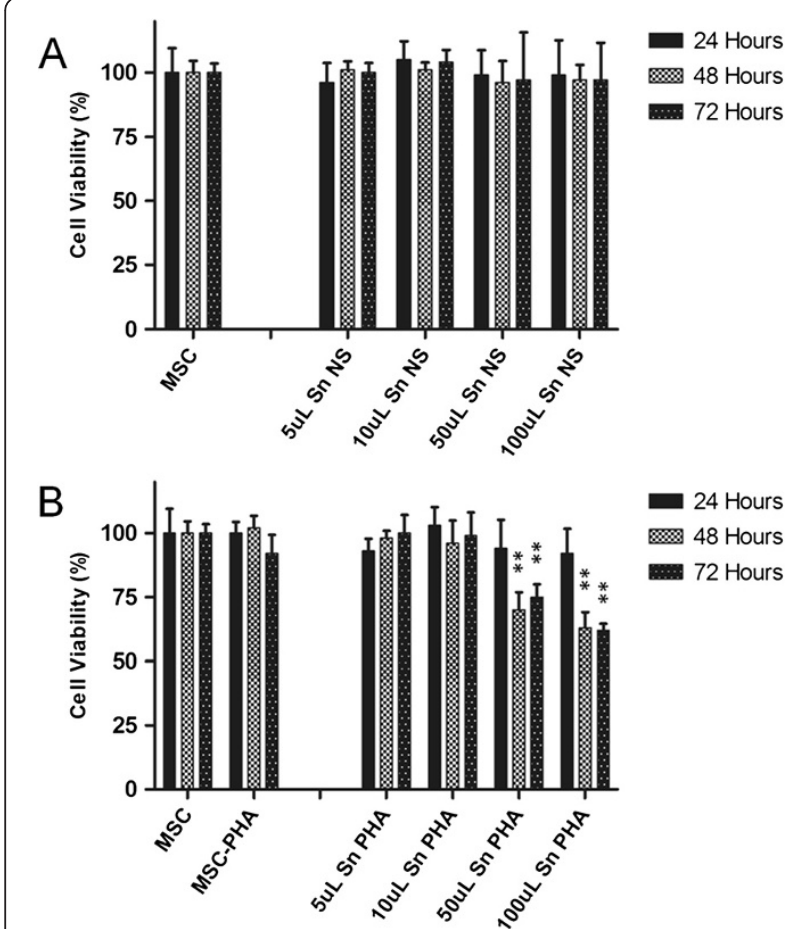

Figure 6 MTT cell viability assay. Cell viability was assessed after MSC treatment with the indicated dose of lymphocyte CM. (A) CM from unstimulated PBMCs; (B) CM from PHA-stimulated PBMCs. Results were analyzed and expressed as percentage of the control (untreated MSCs) and presented as mean values \pm standard deviations of the three independent experiments performed in duplicate. Statistical analysis was performed using Student's t test $\left({ }^{*} P<0.05\right.$; ${ }^{* *} P$ <0.01). CM, conditioned medium; MSCs, mesenchymal stem cells; MTT, (3-(4,5-dimethylthiazol-2-yl)-2,5-diphenyltetrazolium bromide; PBMCs, peripheral blood mononuclear cells; PHA, phytohemagglutinin.

obtained with the sulforhodamine colorimetric B assay, which is based on the cellular protein content [20]. Also, in our experience, the impedance changes were paralleled by similar changes in the MTT assay, confirming that in our setting electric impedance correlates well with the number of living cells in the well.

We showed that pre-activated lymphocytes are able to inhibit the growth of bone marrow-derived MSCs. It should be noted that non-stimulated lymphocytes did not significantly affect the growth of MSCs. In contrast, pre-stimulated lymphocytes induced a complete inhibition of MSC growth, even at the lowest lymphocyte: MSC ratios. Moreover, the impedance-based method allowed us to track the kinetics of MSC growth; while higher numbers of lymphocytes produced a dramatic and rapid effect on the growth of MSCs, lower numbers had a slower but progressive effect, which led at the end to the same level of inhibition of MSC growth. This phenomenon probably indicates that stimulated lymphocytes continue their activation and proliferation regardless of MSC presence.

Since it has been shown that MSCs can be recognized and killed by activated NK [15], we hypothesized that the effect of activated lymphocytes on MSCs could be mostly due to the presence of activated NK cells. In fact, we showed that MSCs are not able to trigger NK degranulation compared to a conventional tumor cell line. Moreover, an inhibitory effect on MSCs was maintained by NK-depleted lymphocytes and similar results were obtained as well with purified $\mathrm{CD} 4^{+}$or $\mathrm{CD}^{+}$subsets. Yet, resting lymphocytes had little effect, thus indicating that activation was necessary to induce maximal inhibition of MSC growth. To evaluate whether the inhibitory effect is due to direct contact between MSCs and lymphocytes or to soluble mediators, CM from activated PBMCs was added to MSCs at different dilutions. Indeed, CM inhibited MSC growth in a dose-dependent manner, suggesting that inflammatory cytokines or other mediators released from activated lymphocytes may have a noxious effect on MSCs. Notably, only higher concentrations of $\mathrm{CM}$ were able to completely block MSC growth, while only a transient effect was obtained with a progressive dilution of CM. Although the growth of IFN- $\gamma$-treated MSCs was only partially affected by pre-activated lymphocytes, it was still inhibited by the addition of CM at certain concentrations. In other words, it is reasonable to state that interferon treatment is effective in potentiating the immunosuppressive action of MSCs, giving them the chance to block the production of lymphocytic mediators that, conversely, can affect the growth of MSCs.

It is noteworthy that a noxious effect of inflammatory cytokines on MSCs has recently been independently demonstrated by different authors. Freytes and collaborators demonstrated that M1 macrophages, producing IL-1 $\beta$, IL-6, TNF- $\alpha$ and IFN- $\gamma$, inhibited the growth of MSC in vitro under certain conditions [20]. Furthermore, Liu and collaborators showed that TNF- $\alpha$ and IFN- $\gamma$ derived from activated lymphocytes are able to block MSC-based bone regeneration [21].

In our setting, TNF- $\alpha$ showed only a limited noxious effect on MSCs when used at supraphysiological concentrations and, thus, it is not likely that it is the main substance responsible for the inhibitory effect of $\mathrm{CM}$. Other biologically active compounds, common to different immune cells, may, of course, play a role. Among these molecules, we could consider nucleotides, such as ATP and ADP, or tryptophan metabolites, known for their immune-regulatory properties. Actually, tryptophan metabolites have been implicated in the immunosuppressive mechanisms of MSCs and, therefore, it is unlikely that they can also affect the viability of MSCs. We, thus, chose to test the action of ATP in our model. We demonstrated that the addition of ATP (to a final concentration 
of $1 \mu \mathrm{M}$ and $10 \mu \mathrm{M}$ ) resulted in changes of MSC CI, similar to those obtained with $\mathrm{CM}$ derived from pre-activated lymphocytes. This novel finding opened the way to other experimental analysis to be performed to clarify the role of nucleotides on MSCs.

The fact that soluble mediators from activated lymphocytes can affect the growth of MSCs raises concern about the use of MSC-based therapies in conditions characterized by ongoing immune activation. Based on these results, we can assume that MSCs may survive briefly and exert limited effects when infused in patients with strong immune activation. In addition, it could be reasonable to hypothesize that MSCs may last longer when infused as a prophylactic treatment before the occurrence of severe immune activation. In fact, MSCs can survive longer in mice, when infused just after hematopoietic stem cell transplantation (HSCT) [22]. However, several reports suggest a role for stimulated lymphocytes in activating the immunosuppressive properties of MSCs [23-25]. Thus, it seems reasonable to hypothesize that the efficacy of MSC as an immunosuppressant in vivo depends upon several factors including: the activation state of lymphocytes, the concentration of inflammatory cytokines in the microenvironment; the ratio between MSCs and lymphocytes; and the licensing of MSCs [26]. As a consequence, the outcome of the struggle between activated lymphocytes and MSCs in vivo cannot be easily predicted by preclinical studies in vitro or in animal models.

Further studies should investigate how different lymphocyte mediators can affect MSC growth.

In addition, in vivo studies are needed to track the effect of the immune activation status on MSC survival.

\section{Conclusions}

In conclusion, strategies aimed at activating MSCs and neutralizing specific mediators released by activated lymphocytes can offer new perspectives for immunomodulatory cell therapies. However, further studies are needed to understand the real impact of lymphocyte-derived mediators on the survival of MSCs infused in vivo.

\section{Additional file}

Additional file 1: Figure A1. Impedance profiles of MSCs (400 cells/well) treated with resting or CD3/28-activated PBMC CM. MSCs were stimulated with CM from unstimulated lymphocytes (A) and from lymphocytes stimulated with anti CD3/28 beads (B). The CM was added to MSCs (400/well) at three different dilutions after about 72 hours of culture. The experiment was conducted in triplicate. Figure $\mathbf{A} \mathbf{2}$. Impedance profile of licensed MSCs in co-culture with PBMCs. Resting (A) or PHA-pre-activated (B) PBMCs were added at four different ratios to MSCs (400/well) pre-treated with IFN- $\gamma$. The experiment was conducted in triplicate. Figure $\mathbf{A} 3$. Impedance profile of licensed MSCs treated with PBMC CM resting (A) or pre-activated (B) lymphocyte CM was added at four different dilutions to MSCs (400/well) pre-treated with IFN- $\gamma$. The experiment was conducted in triplicate.

\section{Abbreviations}

$\mathrm{Cl}$ : cell index; CM: conditioned medium; DMSO: dimethyl sulfoxide; GvHD: graft versus host disease; HLA-l: class I human leukocyte antigen; HSCT: hematopoietic stem cell transplantation; IFN- $\gamma$ : interferon- $\gamma ;$ IL: interleuken; MSC: mesenchymal stromal cell; MTT: 3-(4,5-dimethylthiazol-2-yl)2,5-diphenyltetrazolium bromide; NK: natural killer; PBMC: peripheral blood mononuclear cell; PHA: phytohemagglutinin.

\section{Competing interests}

The authors declare they have no competing interests.

\section{Authors' contributions}

EV designed the experimental plan, performed analysis of results and wrote the manuscript. CL performed additional experiments to review the manuscript and also contributed to the writing of the revised manuscript. SC produced GMP mesenchymal cells, discussed results and corrected the manuscript draft. EP performed the immunologic analyses, discussed the results and corrected the draft. GG supervised the production of mesenchymal cells and discussed the experimental plan. EB discussed results and conclusions of the work. AT is responsible for the project and supervised the entire work. All authors read and approved the final manuscript.

\section{Acknowledgements}

The work was funded by research grant \# 32/11 of the IRCCS Burlo Garofolo. We would like to thank the Laboratory of Cell Therapy 'Stefano Verri' in Monza for their invaluable support in continuously providing us with GMP-grade bone-marrow-derived MSC.

\section{Author details}

'Department of Pediatrics, Institute of Maternal and Child Health IRCCS Burlo Garofolo, Via dell'Istria 65/1, 34137 Trieste, Italy. ${ }^{2}$ M. Tettamanti Research Center, Pediatric Clinic University of Milano Bicocca, Monza, Italy. ${ }^{3}$ Cell Therapy Laboratory "Stefano Verri", San Gerardo Hospital, Monza, Italy.

Received: 9 April 2013 Revised: 8 October 2013

Accepted: 20 December 2013 Published: 9 January 2014

\section{References}

1. Friedenstein AJ, Chailakhjan RK, Lalykina KS: The development of fibroblast colonies in monolayer cultures of guinea-pig bone marrow and spleen cells. Cell Tissue Kinet 1970, 3:393-403.

2. Wang HS, Hung SC, Peng ST, Huang CC, Wei HM, Guo YJ: Mesenchymal stem cells in the Wharton's jelly of the human umbilical cord. Stem Cells 2004, 22:1330-1337.

3. Castro-Malaspina H, Gay RE, Resnick G, Kapoor N, Meyers P, Chiarieri D, McKenzie S, Broxmeyer HE, Moore MA: Characterization of human bone marrow fibroblast colony-forming cells (CFU-F) and their progeny. Blood 1980, 56:289-301.

4. Caplan Al: Mesenchymal stem cells. J Orthop Res 1991, 9:641-650.

5. Zuk PA, Zhu M, Mizuno H, Huang J, Futrell JW, Katz AJ, Benhaim P, Lorenz HP, Hedrick MH: Multilineage cells from human adipose tissue: implications for cell-based therapies. Tissue Eng 2001, 7:211-228.

6. Jankowski RJ, Deasy BM, Huard J: Muscle-derived stem cells. Gene Ther 2002, 9:642-647.

7. Dominici M, Le Blanc K, Mueller I, Slaper-Cortenbach I, Marini F, Krause D, Deans R, Keating A, Prockop D, Horwitz E: Minimal criteria for defining multipotent mesenchymal stromal cells. The International Society for Cellular Therapy position statement. Cytotherapy 2006, 8:315-317.

8. Le Blanc K, Frassoni F, Ball L, Locatelli F, Roelofs H, Lewis I, Lanino E, Sundberg B, Bernardo ME, Remberger M, Dini G, Egeler RM, Bacigalupo A, Fibbe W, Ringdén O: Mesenchymal stem cells for treatment of steroidresistant, severe, acute graft-versus-host disease: a phase II study. Lancet 2008, 371:1579-1586

9. Arima N, Nakamura F, Fukunaga A, Hirata H, Machida H, Kouno S, Ohgushi H: Single intra-arterial injection of mesenchymal stromal cells for treatment of steroid-refractory acute graft-versus-host disease: a pilot study. Cytotherapy 2010, 12:265-268.

10. Kebriaei P, Isola L, Bahceci E, Holland K, Rowley S, McGuirk J, Devetten M, Jansen J, Herzig R, Schuster M, Monroy R, Uberti J: Adult human 
mesenchymal stem cells added to corticosteroid therapy for the treatment of acute graft-versus-host disease. Biol Blood Marrow Transplant 2009, 15:804-811.

11. Lucchini G, Introna M, Dander E, Rovelli A, Balduzzi A, Bonanomi S, Salvadè A, Capelli C, Belotti D, Gaipa G, Perseghin P, Vinci P, Lanino E, Chiusolo P, Orofino MG, Marktel S, Golay J, Rambaldi A, Biondi A, D'Amico G, Biagi E: Platelet-lysate-expanded mesenchymal stromal cells as a salvage therapy for severe resistant graft-versus-host disease in a pediatric population. Biol Blood Marrow Transplant 2010, 16:1293-1301.

12. Jacobs SA, Pinxteren J, Roobrouck VD, Luyckx A, Van't Hof W, Deans R, Verfaillie CM, Waer M, Billiau AD, Van Gool SW: Human multipotent adult progenitor cells are non-immunogenic and exert potent immunomodulatory effects on alloreactive $T$ cell responses. Cell Transplant 2012, 22:1915-1928.

13. Hoogduijn MJ, Roemeling-van Rhijn M, Korevaar SS, Engela AU, Weimar W, Baan CC: Immunological aspects of allogeneic and autologous mesenchymal stem cell therapies. Hum Gene Ther 2011, 22:1587-1591.

14. Morandi F, Raffaghello L, Bianchi G, Meloni F, Salis A, Millo E, Ferrone S, Barnaba $V$, Pistoia $V$ : Immunogenicity of human mesenchymal stem cells in HLA-class I-restricted T-cell responses against viral or tumorassociated antigens. Stem Cells 2008, 26:1275-1287.

15. Spaggiari GM, Capobianco A, Becchetti S, Mingari MC, Moretta L: Mesenchymal stem cell-natural killer cell interactions: evidence that activated NK cells are capable of killing MSCs, whereas MSCs can inhibit IL-2-induced NK-cell proliferation. Blood 2006, 107:1484-1490.

16. Spaggiari GM, Capobianco A, Abdelrazik H, Becchetti F, Mingari MC, Moretta L: Mesenchymal stem cells inhibit natural killer-cell proliferation, cytotoxicity, and cytokine production: role of indoleamine 2,3-dioxygenase and prostaglandin E2. Blood 2008, 111:1327-1333.

17. Valencic E, Piscianz E, Andolina M, Ventura A, Tommasini A: The immunosuppressive effect of Wharton's jelly stromal cells depends on the timing of their licensing and on lymphocyte activation. Cytotherapy 2010, 12:154-160.

18. Angstmann M, Brinkmann I, Bieback K, Breitkreutz D, Maercker C: Monitoring human mesenchymal stromal cell differentiation by electrochemical impedance sensing. Cytotherapy 2011 13:1074-1089

19. Bryceson $Y T$, Pende D, Maul-Pavicic A, Gilmour KC, Ufheil H, Vraetz T, Chiang SC, Marcenaro S, Meazza R, Bondzio I, Walshe D, Janka G, Lehmberg K, Beutel K, zur Stadt U, Binder N, Arico M, Moretta L Henter Jl, Ehl S: A prospective evaluation of degranulation assays in the rapid diagnosis of familial hemophagocytic syndromes. Blood 2012, 119:2754-2763.

20. Freytes DO, Kang JW, Marcos I, Vunjak-Novakovic G: Macrophages modulate the viability and growth of human mesenchymal stem cells. J Cell Biochem 2012, 114:220-229.

21. Liu Y, Wang L, Kikuiri T, Akiyama K, Chen C, Xu X, Yang R, Chen W, Wang S, Shi S: Mesenchymal stem cell-based tissue regeneration is governed by recipient T lymphocytes via IFN-gamma and TNF-alpha. Nat Med 2011 17:1594-1601.

22. Sudres M, Norol F, Trenado A, Gregoire S, Charlotte F, Levacher B, Lataillade $J$ J, Bourin P, Holy X, Vernant JP, Klatzmann D, Cohen JL: Bone marrow mesenchymal stem cells suppress lymphocyte proliferation in vitro but fail to prevent graft-versus-host disease in mice. J Immunol 2006, 176:7761-7767.

23. Renner P, Eggenhofer E, Rosenauer A, Popp FC, Steinmann JF, Slowik P, Geissler EK, Piso P, Schlitt HJ, Dahlke MH: Mesenchymal stem cells require a sufficient, ongoing immune response to exert their immunosuppressive function. Transp/ Proc 2009, 41:2607-2611.

24. Chang CJ, Yen ML, Chen YC, Chien CC, Huang HI, Bai CH, Yen BL: Placenta-derived multipotent cells exhibit immunosuppressive properties that are enhanced in the presence of interferon-gamma. Stem Cells 2006, 24:2466-2477.
25. Ryan JM, Barry F, Murphy JM, Mahon BP: Interferon-gamma does not break, but promotes the immunosuppressive capacity of adult human mesenchymal stem cells. Clin Exp Immunol 2007, 149:353-363.

26. Wang L, Zhao Y, Shi S: Interplay between mesenchymal stem cells and lymphocytes: implications for immunotherapy and tissue regeneration. J Dent Res 2012, 91:1003-1010.

doi:10.1186/scrt392

Cite this article as: Valencic et al.: Inhibition of mesenchymal stromal cells by pre-activated lymphocytes and their culture media. Stem Cell Research \& Therapy 2014 5:3.

\section{Submit your next manuscript to BioMed Central and take full advantage of:}

- Convenient online submission

- Thorough peer review

- No space constraints or color figure charges

- Immediate publication on acceptance

- Inclusion in PubMed, CAS, Scopus and Google Scholar

- Research which is freely available for redistribution

Submit your manuscript at www.biomedcentral.com/submit
C Biomed Central 